\title{
Dynamical model of coherent circularly polarized optical pulse interactions with two-level quantum systems
}

\author{
G. Slavcheva* and O. Hess \\ Advanced Technology Institute, School of Electronics and Physical Sciences, University of Surrey, \\ Guildford GU2 7XH, Surrey, United Kingdom \\ (Received 1 August 2005; published 3 November 2005)
}

\begin{abstract}
We propose and develop a method for theoretical description of circularly (elliptically) polarized optical pulse resonant coherent interactions with two-level atoms. The method is based on the time-evolution equations of a two-level quantum system in the presence of a time-dependent dipole perturbation for electric dipole transitions between states with total angular-momentum projection difference $\left(\Delta J_{z}= \pm 1\right)$ excited by a circularly polarized electromagnetic field [Feynman et al., J. Appl. Phys. 28, 49 (1957)]. The adopted real-vector representation approach allows for coupling with the vectorial Maxwell's equations for the optical wave propagation and thus the resulting Maxwell pseudospin equations can be numerically solved in the time domain without any approximations. The model permits a more exact study of the ultrafast coherent pulse propagation effects taking into account the vector nature of the electromagnetic field and hence the polarization state of the optical excitation. We demonstrate self-induced transparency effects and formation of polarized solitons. The model represents a qualitative extension of the well-known optical Maxwell-Bloch equations valid for linearly polarized light and a tool for studying coherent quantum control mechanisms.
\end{abstract}

DOI: 10.1103/PhysRevA.72.053804

PACS number(s): 42.50.Ct, 42.50.Md, 42.50.Gy, 42.50.Nn

\section{INTRODUCTION}

With the view of achieving quantum coherent control and manipulation of spin-polarized states in semiconductor nanostructures recently there has been a significant interest in circularly (elliptically) polarized optical pulse excitations due to their ability to transfer angular-momentum and drive a system to a particular angular-momentum state. A necessary condition for quantum coherence is the use of sufficiently short optical pulses so that they can interact with the quantum system before it can be affected by its environment. The achievement of control and manipulation of the dynamics of a single (or a few) electron spins (quantum bits) in semiconductor nanostructures is of key importance toward the challenging goal of realization of quantum computation. Recent research [2] has demonstrated that semiconductor quantum dots might be strong candidates for the realization of spin quantum bits. This is due to their atomiclike electronic structure which suppresses coupling of the spin to the solid-state quantum environment and thus impedes the spin decoherence processes. In this respect, the analogy with the discrete multilevel system becomes more relevant and permits modeling of the quantum-dot coherent dynamics on the basis of the Maxwell pseudospin equations [3].

It is well known that in the coherent regime the time evolution of the two-level quantum system under external dipole coupling perturbation can be described by the coherent optical Bloch equations (see, e.g., [4]). These equations, however, have been derived under the assumptions of a linearly polarized optical excitation for which the selection rules $\Delta J_{z}=0$ apply. The optical transitions between the two levels are induced by the dipole interaction which is given by

*Electronic address: G.Slavcheva@surrey.ac.uk the scalar product $\vec{\mu} \cdot \vec{E}(t), \vec{\mu}$ being the dipole moment. However, the vectorial character of the electric field $\vec{E}(t)$ is completely ignored and only $\mu E(t)$ is considered.

The optical Bloch equations with linear polarization coupled with the vector Maxwell's equations have been successfully applied for description of ultrashort pulse propagation in nonlinear media $[5,6]$ and for demonstration of selfinduced transparency effects and soliton propagation in twolevel atomic absorbing or amplifying media in one dimension (1D) [7].

However, the selection rules for the excitonic transitions in bulk semiconductors, and in particular in semiconductors with reduced dimensionality, require $\Delta J_{z}= \pm 1$. These transitions are excited by elliptically (or circularly) polarized light and can no longer be described by a single scalar interaction, as has been already pointed out by Feynman et al. [1]. This justifies the need for a model of coherent dynamics accounting for the polarization state of the applied electromagnetic field. In what follows, we shall be interested in circularly polarized pulse propagation and interactions, inducing the above type of optically allowed (dipole-active) transitions between the quantum states.

In this paper we develop a general formalism and propose a numerical model for description of polarized ultrashort optical pulse interactions with a two-level quantum system. The polarized ultrashort optical pulse propagation and interactions with the discrete quantum-level system are treated within the semiclassical approach. The optical field is calculated self-consistently from the vectorial Maxwell equations coupled via macroscopic polarization to the time-evolution equations of the quantum system in terms of the real pseudospin (coherence) vector [1]. The latter represents a special case $(N=2)$ of the generalized pseudospin formalism for an $N$-level quantum system $[8,9]$. The system is solved numerically in the time domain without invoking the standard ap- 


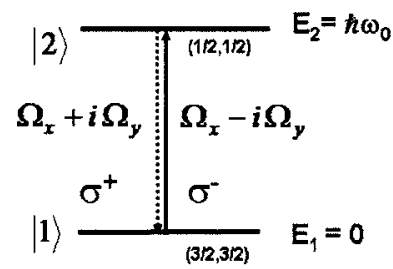

(a)

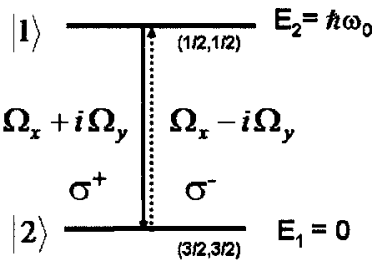

(b)

FIG. 1. Energy-level diagram of a two-level quantum system with $\Delta J_{z}= \pm 1$ dipole-allowed transitions and energy separation $\hbar \omega_{0}$. States are labeled by pairs of total angular momentum and its projection along the propagation $z$ axis. (a) The resonant medium modeled by an ensemble of two-level systems is assumed to be initially absorbing (all atoms in their ground states). The population is driven into the upper excited level $|2\rangle$ by left-circularly-polarized optical field $\left(\sigma^{-}\right)$and returned back to its initial state $|1\rangle$ by rightcircularly-polarized optical field $\left(\sigma^{+}\right)$. (b) The system is amplifying (all atoms in the excited state). The population is driven from the initial excited level $|1\rangle$ to the final ground state $|2\rangle$ by rightcircularly-polarized optical field $\left(\sigma^{+}\right)$and returned back to the excited state by left-circularly-polarized optical field $\left(\sigma^{-}\right)$.

proximations [such as the slowly-varying-envelope approximation (SVEA) and rotating-wave approximation (RWA)] by an iterative predictor-corrector finite-difference time-domain method. This allows for a rigorous treatment of optical excitations on an ultrashort time scale (including few-cycle optical pulses).

The paper is organized as follows. In Sec. II we describe the theoretical background behind the model. In Sec. III we give details of the numerical implementation and summarize the results from the numerical simulations in the linear regime, demonstrating selective excitation or deexcitation of the quantum system by each sense of the optical wave polarization when the pulse propagates in an absorbing or amplifying medium represented by an ensemble of two-level systems. In Sec. IV we give the simulation results for highintensity optical pulses exhibiting self-induced transparency and demonstrate numerically the existence of polarized solitons. Finally, conclusions are drawn in Sec. V.

\section{THEORETICAL MODEL}

Consider a plane electromagnetic wave propagating along the $z$ direction, elliptically polarized in a perpendicular plane to $z$. During its propagation, the optical wave interacts with the two-level medium schematically shown in Fig. 1, thus inducing a polarization along the $x$ and $y$ directions. The one-dimensional Maxwell equations in vector form then read

$$
\begin{gathered}
\frac{\partial H_{x}(z, t)}{\partial t}=-\frac{1}{\mu} \frac{\partial E_{y}(z, t)}{\partial z}, \\
\frac{\partial H_{y}(z, t)}{\partial t}=-\frac{1}{\mu} \frac{\partial E_{x}(z, t)}{\partial z}, \\
\frac{\partial E_{x}(z, t)}{\partial t}=-\frac{1}{\varepsilon} \frac{\partial H_{y}(z, t)}{\partial z}-\frac{1}{\varepsilon} \frac{\partial P_{x}(z, t)}{\partial t},
\end{gathered}
$$

$$
\frac{\partial E_{y}(z, t)}{\partial t}=\frac{1}{\varepsilon} \frac{\partial H_{x}(z, t)}{\partial z}-\frac{1}{\varepsilon} \frac{\partial P_{y}(z, t)}{\partial t} .
$$

Let us construct phenomenologically a total Hamiltonian corresponding to the energy-level scheme in Fig. 1. We shall consider more specifically an absorbing medium, i.e., all the atoms initially are in their ground states (the case of an amplifying medium is considered analogously). The system Hamiltonian for a two-level system is a $2 \times 2$ matrix and in the presence of dipole coupling perturbation is a sum of the unperturbed (in the absence of an electromagnetic interaction) and dipole interaction perturbation Hamiltonians:

$$
\hat{H}=\hat{H}_{0}+\hat{H}_{i n t}=\hbar\left(\begin{array}{cc}
0 & -\frac{1}{2}\left(\Omega_{x}-i \Omega_{y}\right) \\
-\frac{1}{2}\left(\Omega_{x}+i \Omega_{y}\right) & \omega_{0}
\end{array}\right) .
$$

In constructing the perturbation Hamiltonian we assume that the transition between the ground state $|1\rangle$ and the excited state $|2\rangle$ [Fig. 1(a)] is induced by generally left-elliptically polarized light $\sigma^{-}$, which we take to be $\Omega_{x}-i \Omega_{y}$, while the opposite virtual transition is excited by right-elliptically polarized light $\sigma^{+}, \Omega_{x}+i \Omega_{y}$, where we have introduced Rabi frequencies associated with the electric field components along the $x$ and $y$ axes, according to

$$
\begin{aligned}
& \Omega_{x}=\frac{\wp}{\hbar} E_{x}, \\
& \Omega_{y}=\frac{\wp}{\hbar} E_{y}
\end{aligned}
$$

with dipole coupling coefficient given by $\wp=e q_{0}, q_{0}$ being the typical separation between the charges in a dipole (e.g., atomic or exciton Bohr radius); $\omega_{0}$ is the resonant angular frequency associated with a transition between the two levels.

We shall show that the elements of the so-constructed Hamiltonian coincide with the matrix elements derived in [1] in the case of electric $\Delta J_{z}= \pm 1$ transitions. In what follows, we shall use the generalized pseudospin formalism introduced in [9] to derive the time-evolution real coherence (pseudospin) vector equations of the two-level system. We shall show the equivalence of the real three-vector Feynman approach and the pseudospin equations. Using the expressions derived in [9] for the coefficients in the expansions of the density matrix and the system Hamiltonian in terms of the generators of the $\mathrm{SU}(2)$ algebra $\hat{\lambda}_{j}$, the real torque vector components are calculated from

$$
\gamma_{j}(t)=\frac{1}{\hbar} \operatorname{Tr}\left[\hat{H}(t) \hat{\lambda_{j}}\right], \quad j=1,2,3
$$

where $\hat{\lambda}_{j}$ are the Pauli matrices given by

$$
\hat{\lambda}_{1}=\left(\begin{array}{ll}
0 & 1 \\
1 & 0
\end{array}\right), \quad \hat{\lambda}_{2}=\left(\begin{array}{cc}
0 & -i \\
i & 0
\end{array}\right), \quad \hat{\lambda}_{3}=\left(\begin{array}{cc}
1 & 0 \\
0 & -1
\end{array}\right),
$$

giving

$$
\vec{\gamma}=\left(-\boldsymbol{\Omega}_{x},-\boldsymbol{\Omega}_{y},-\omega_{0}\right)
$$


It has been shown [9] that the time evolution of the density matrix can be expressed in terms of the evolution of an $\left(N^{2}-1\right)$-dimensional real coherence (pseudospin) vector $S_{j}$ satisfying the following equation of motion:

$$
\dot{S}_{i}=f_{i j k} \gamma_{j} S_{k}, \quad i, j, k=1,2,3 .
$$

Substituting (2.10) and the nonvanishing components of the fully antisymmetric tensor $f$ of the structure constants of the $\mathrm{SU}(2)$ group, given by

$$
\begin{aligned}
& f_{123}=f_{231}=f_{312}=1, \\
& f_{132}=f_{213}=f_{321}=-1
\end{aligned}
$$

in the above equations, we get the equations of motion of the real pseudospin three-vector:

$$
\begin{gathered}
\frac{\partial S_{1}}{\partial t}=\omega_{0} S_{2}-\Omega_{y} S_{3}-\frac{S_{1}}{T_{2}}, \\
\frac{\partial S_{2}}{\partial t}=-\omega_{0} S_{1}+\Omega_{x} S_{3}-\frac{S_{2}}{T_{2}}, \\
\frac{\partial S_{3}}{\partial t}=\Omega_{y} S_{1}-\Omega_{x} S_{2}-\frac{\left(S_{3}-S_{30}\right)}{T_{1}} .
\end{gathered}
$$

Above we have introduced damping in the system by inclusion of the phenomenological relaxation times $T_{1}$ and $T_{2}$, which have the usual meaning of population relaxation and dephasing time, respectively, and $S_{30}$ is the initial population profile. $S_{30}=-1$ initially set corresponds to all resonant dipoles in their ground states and therefore the modeled medium will be absorbing; $S_{30}=+1$ corresponds to all dipoles initially excited in the upper energy level describing amplifying (gain) medium.

The above system coincides with the equation of motion derived by Feynman et al. in the case of electric $\Delta J_{z}= \pm 1$ dipole transitions $[1]$ :

$$
\frac{d \mathbf{S}}{d t}=\gamma \times \mathbf{S},
$$

which is of the well-known form of precession of a classical gyromagnet in a magnetic field.

The macroscopic polarization density induced in the medium is given by

$$
\mathbf{P}=-e N_{a} \operatorname{Tr}(\hat{\rho} \hat{Q})
$$

where $\hat{\rho}$ is the density matrix, $\hat{Q}$ is the local displacement operator whose expectation value gives the local displacement from equilibrium vector $\mathbf{q}$ and $N_{a}$ is the resonant dipole density. During its propagation the optical wave induces a polarization along the $x$ and $y$ axes, therefore the local displacement operator can be split into two orthogonal components

$$
\hat{Q}_{x}=\frac{q_{0}}{2} \hat{\lambda}_{1},
$$

$$
\hat{Q}_{y}=\frac{q_{0}}{2} \hat{\lambda}_{2}
$$

where $\lambda_{1}, \lambda_{2}$ are given by Eq. (2.9). It is easy to show that with this expansion and an electric field vector within the $x$ $-y$ plane, we can recover the dipole interaction Hamiltonian from Eq. (2.5). Using Eq. (2.18) we get

$$
\begin{aligned}
& P_{x}=-\frac{1}{2} \wp N_{a} S_{1}, \\
& P_{y}=-\frac{1}{2} \wp N_{a} S_{2} .
\end{aligned}
$$

Thus we end up with the time-evolution equations of the two-level quantum system (2.14)-(2.16) coupled to the Maxwell curl equations (2.1)-(2.4) via the polarization (2.21) and (2.22) acting as a source term in Maxwell's equations.

\section{NUMERICAL RESULTS}

We consider the Goursat-type inital boundary value problem [10] for the solution in the time domain of the above first-order system of differential equations. The problem is well posed if the whole time history of the pulse excitation is given at a certain characteristic of the medium, e.g., at the left boundary $z=0$. In order to model an elliptically (circularly) polarized optical pulse we excite simultaneously two orthogonal linearly polarized pulses, one time delayed with respect to the other by $\pi / 2$ at the left boundary. We adopt the following convention for the ellipticity sense. The optical pulse given by

$$
\begin{gathered}
E_{x}(z=0, t)=\widetilde{E}_{x}(t) \cos \left(\omega_{0} t\right), \\
E_{y}(z=0, t)=-\widetilde{E}_{y}(t) \sin \left(\omega_{0} t\right)
\end{gathered}
$$

will be associated with left-circularly-polarized light $\sigma^{-}$, while the right-circularly-polarized light $\sigma^{+}$will be simulated by

$$
\begin{aligned}
& E_{x}(z=0, t)=\widetilde{E}_{x}(t) \cos \left(\omega_{0} t\right), \\
& E_{y}(z=0, t)=\widetilde{E}_{y}(t) \sin \left(\omega_{0} t\right),
\end{aligned}
$$

where $\omega_{0}$ is the pulse carrier frequency at resonance with the two-level system and the envelopes $\widetilde{E}_{x}(t)$ and $\widetilde{E}_{y}(t)$ determine the specific shape (e.g., hyperbolic secant or Gaussian) of the pulse:

$$
\begin{aligned}
\tilde{E}_{x, y}(t) & \\
& =\left\{\begin{array}{l}
E_{0} \operatorname{sech}(10 \Gamma), \\
E_{0} \exp \left[-\left(t-t_{0}\right)^{2} / t_{\text {decay }}^{2}\right] .
\end{array} \quad \Gamma=\left[t-\left(T_{p} / 2\right)\right] /\left(T_{p} / 2\right),\right.
\end{aligned}
$$

In the above equation $E_{0}$ is the initial field amplitude and $T_{p}$ is the temporal pulse width.

Following [7], we introduce new variables according to 


$$
\begin{gathered}
S_{1}=e^{-t / T_{2}} u_{1}, \\
S_{2}=e^{-t / T_{2}} u_{2}, \\
S_{3}=S_{30}+e^{-t / T_{1}} u_{3}
\end{gathered}
$$

and recast the pseudospin system into

$$
\begin{gathered}
\frac{\partial u_{1}}{\partial t}=\omega_{0} u_{2}-D(t) E_{y}-C_{+}(t) E_{y} u_{3}, \\
\frac{\partial u_{2}}{\partial t}=-\omega_{0} u_{1}+D(t) E_{x}+C_{+}(t) E_{x} u_{3}, \\
\frac{\partial u_{3}}{\partial t}=C_{-}(t) E_{y} u_{1}-C_{-}(t) E_{x} u_{2},
\end{gathered}
$$

where we have introduced the following time-dependent coefficients:

$$
\begin{gathered}
C_{ \pm}(t)=\frac{\wp}{\hbar} e^{\mp t\left(1 / T_{1}-1 / T_{2}\right)}, \\
D(t)=\frac{\wp}{\hbar} S_{30} e^{t / T_{2}} .
\end{gathered}
$$

Differentiating (2.21) and (2.22) with respect to time using (2.14)-(2.16), taking advantage of the newly introduced variables, and substituting back in Maxwell's equations (2.3) and (2.4), we get

$$
\begin{gathered}
\frac{\partial H_{x}}{\partial t}=\frac{1}{\mu} \frac{\partial E_{y}}{\partial z} \\
\frac{\partial H_{y}}{\partial t}=-\frac{1}{\mu} \frac{\partial E_{x}}{\partial z} \\
\frac{\partial E_{x}}{\partial t}=-\frac{1}{\varepsilon} \frac{\partial H_{y}}{\partial z}-A(t) u_{1}+B_{1}(t) u_{2}-C_{1} E_{y} S_{30}-C_{2}(t) E_{y} u_{3}, \\
\frac{\partial E_{y}}{\partial t}=\frac{1}{\varepsilon} \frac{\partial H_{x}}{\partial z}-B_{1}(t) u_{1}-A(t) u_{2}+C_{1} E_{x} S_{30}+C_{2}(t) E_{x} u_{3}
\end{gathered}
$$

which together with the pseudospin equations (3.9)-(3.11) comprises the final system of equations describing elliptically polarized pulse interaction with a two-quantum level system. We have introduced above the following coefficients:

$$
\begin{gathered}
A(t)=\frac{\wp N_{a}}{2 \varepsilon T_{2}} e^{-t / T_{2}}, \\
B_{1}(t)=\frac{\wp N_{a} \omega_{0}}{2 \varepsilon} e^{-t / T_{2}}, \\
C_{1}=\frac{\wp^{2} N_{a}}{2 \varepsilon \hbar}
\end{gathered}
$$

$$
C_{2}(t)=\frac{\wp^{2} N_{a}}{2 \varepsilon \hbar} e^{-t / T_{1}}
$$

The linear polarization case can be easily recovered by setting $E_{y}=0$ and $H_{x}=0$ in the above system thus obtaining the common coherent optical Maxwell-Bloch equations (see [7]).

The so-obtained coherent vectorial Maxwell pseudospin equations (3.14)-(3.17) and (3.9)-(3.11) are discretized on a Yee grid using a finite-difference time-domain (FDTD) timestepping algorithm [11] with a predictor-corrector iterative scheme implemented at each time step [7]. The boundary conditions imposed on the left and the right boundaries are perfectly transmitting with a second-order-accuracy difference scheme [6].

In order to demonstrate selective excitation or deexcitation of the two-level system by each sense of circular polarization and the failure of exciting or deexciting it by the opposite sense of circular polarization, we have performed the following numerical experiment. Consider a $15-\mu \mathrm{m}-\mathrm{long}$ simulation domain filled with a resonantly absorbing GaAs medium with refractive index $n=3.3827$ at wavelength $\lambda$ $=1.5 \mu \mathrm{m}$ (the initial population profile is $S_{30}=-1$ ). At the left boundary of the FDTD simulation space two orthogonal Gaussian pulses phase shifted by $\pi / 2$ and given by Eqs. (3.1) and (3.2) with an envelope given by the second line of Eq. (3.5) are launched toward the slab. The carrier frequency of the pulses is tuned in resonance with the atomic transition frequency $\omega_{0}$ corresponding to the wavelength $\lambda=1.5 \mu \mathrm{m}$, and the pulse duration is chosen as $T_{p}=30 \mathrm{fs}$. We shall restrict ourselves to the linear absorption and gain regime; therefore the initial electric field amplitude is chosen to be $E_{0}=1 \mathrm{~V} \mathrm{~m}^{-1}$. The population and polarization relaxation times of the damped resonant dipoles are set to $T_{1}=100 \mathrm{ps}$ and $T_{2}=70 \mathrm{fs}$, respectively. The electric field is sampled as a function of time at two locations $z_{1}$ and $z_{2}$ within the absorbing medium separated by one dielectric wavelength. The complex propagation factors over distance $l=z_{2}-z_{1}=\lambda / n$ for the $E_{x}$ and $E_{y}$ components of the electric field are calculated by taking the Fourier transform of the time traces, according to

$$
e^{i k_{c}\left(z_{2}-z_{1}\right)}=\frac{E_{j}\left(z_{2}, \omega\right)}{E_{j}\left(z_{1}, \omega\right)}, \quad j=x, y,
$$

where the complex wave vector is given by $k_{c}=\beta+i \gamma$. Separating the real and imaginary parts we can obtain the amplification (absorption) factor $e^{-\gamma l}$ and the phase factor $\beta l$. From the former the gain (absorption) coefficient can be calculated and from the latter the atomic phase shift is inferred. These can be compared with the theoretically calculated atomic absorption and gain coefficients and phase shifts from a steadystate solution of the density matrix system for linear polarization [12]. We shall be interested in a quantitative comparison with the linear polarization (LP) absorption and gain coefficients given by 

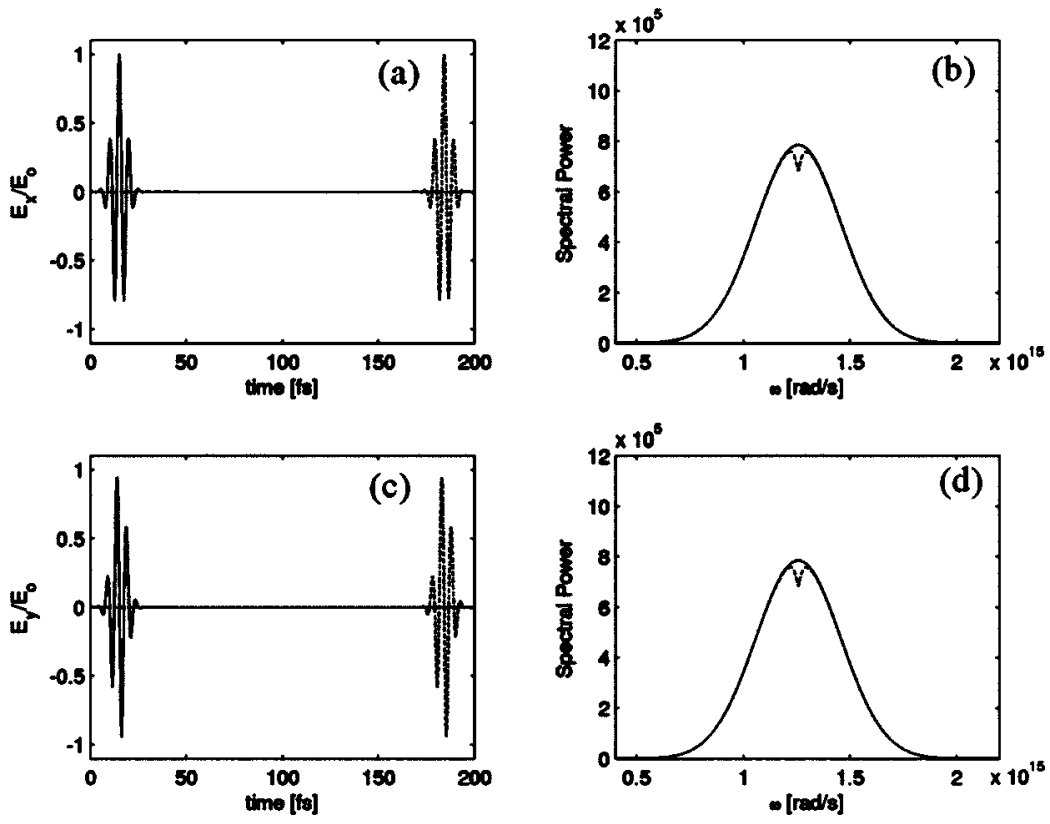

2(c). The optical wave interacts with the two-level system driving the population to the upper state by absorbing the pulse energy. Therefore we clearly observe absorption for both $E_{x}$ and $E_{y}$ components of the electric field in the Fourier spectra plotted in Figs. 2(b) and 2(d).

The absorption coefficients for $E_{x}$ and $E_{y}$ field components are shown in Figs. 3(a) and 3(b) compared with the theoretical result for linear polarization calculated from Eq. (3.23). The absorption coefficient for each $E$ field component of the circularly polarized optical pulse coincides with the absorption for the linear polarization case. In order to prove that only the left-circularly-polarized field excites the twolevel system [Fig. 1(a)] we need to show that an optical wave with an opposite sense of ellipticity fails to excite the system. Therefore we inject in the absorbing medium a rightcircularly-polarized pulse $\sigma^{+}(+,+)$given by Eqs. (3.3) and (3.4) with a Gaussian envelope given by the second line in Eq. (3.5) similar to the previous case. The pulse time evolu-
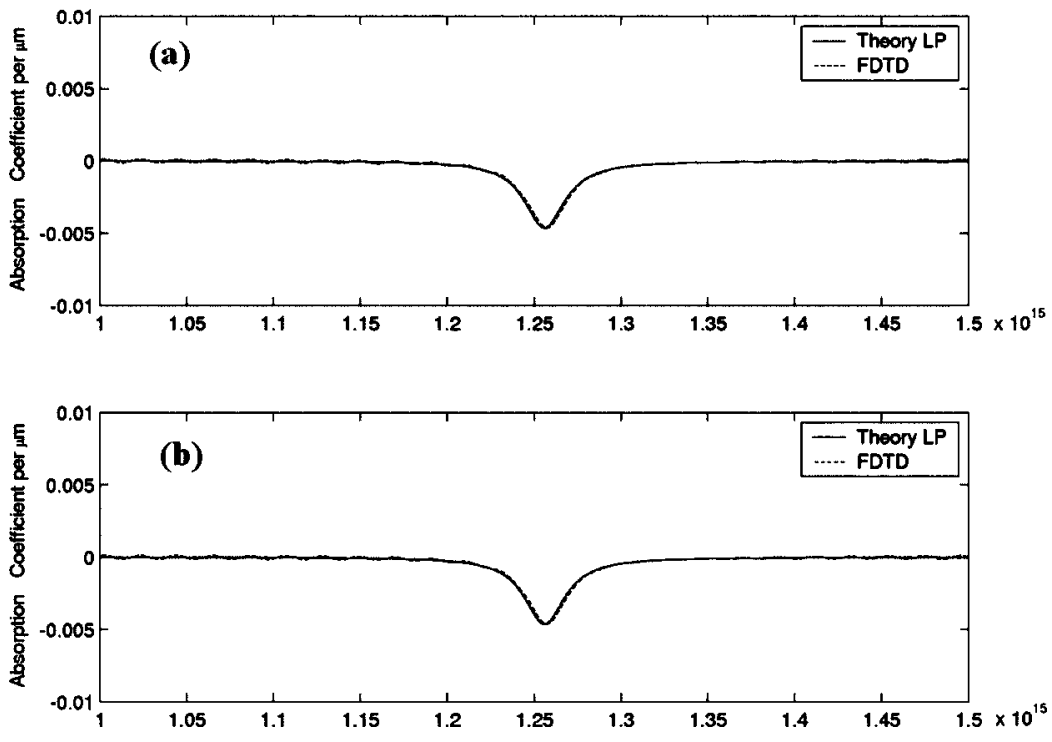

FIG. 3. Absorption coefficient for $E_{x}$ component (a) and $E_{y}$ component (b) of the electric field calculated from the real part of Eq. (3.22) using the FDTD solution of the Maxwell pseudospin system (dashed line) compared with the theoretical absorption coefficient as calculated from the steady-state solution of the Maxwell-Bloch system with linear polarization from Eq. (3.23) (solid line). Excellent agreement is found for both $E$ field components. 

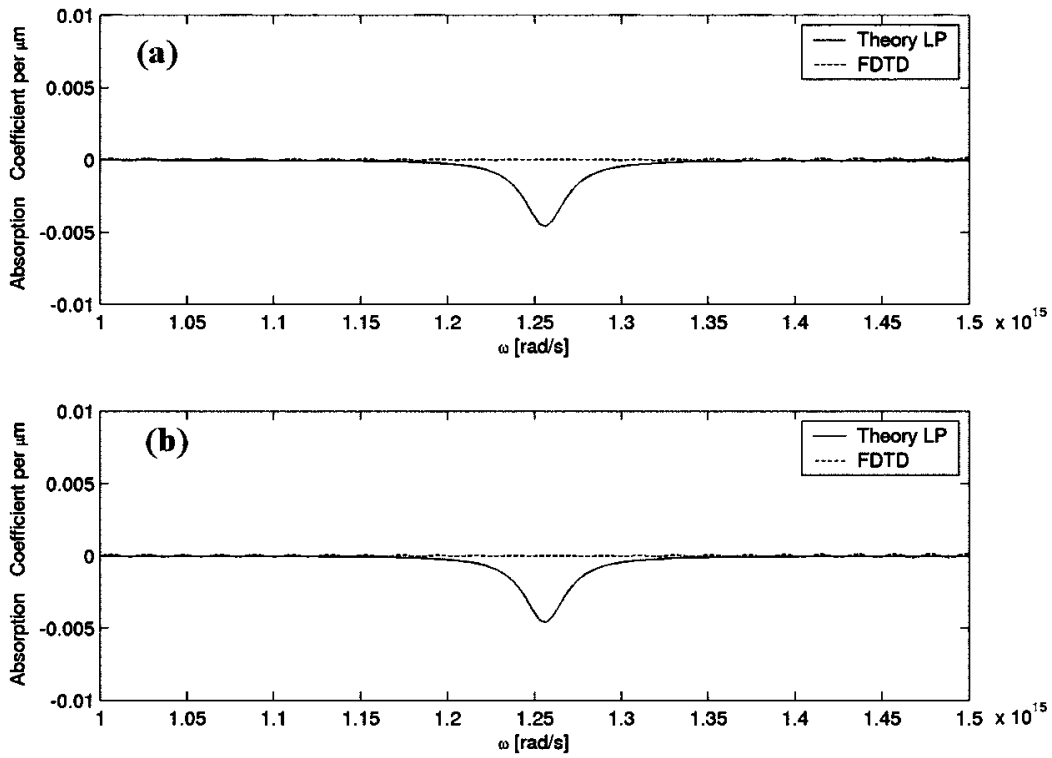

FIG. 4. Absorption coefficient for $E_{x}$ component (a) and $E_{y}$ component (b) of a rightcircularly-polarized optical field $\sigma^{+}(+,+)$compared with the theoretically calculated absorption coefficient for linear polarization showing that the pulse does not interact with the two-level system. tion is sampled again in two locations separated by one dielectric wavelength from which we calculate the absorption coefficient. In this case there is no change in the Fourier spectrum of the pulses indicating absorption. In Fig. 4 we plot the absorption coefficient for each electric field component as inferred from the FDTD calculations compared with the theoretically calculated absorption coefficient corresponding to the linear polarization case for reference.

In order to prove that our model correctly describes the excitation of the two-level system with $\Delta J_{z}= \pm 1$ electric dipole transitions, it is necessary to show that when the system is inverted (the whole population is residing on the upper level) and represents an amplifying (gain) medium the rightcircularly-polarized optical pulse will return the system to its ground state. We consider an initially completely inverted two-level system $\left(S_{30}=+1\right)$. We shall show that by injecting the right-circularly-polarized pulse $\sigma^{+}(+,+)$given by Eqs. (3.3) and (3.4) with a Gaussian envelope and all other pa- rameters kept the same in conformity with the previous two cases, the population is driven into the system ground state. The electric field components of the right-circularlypolarized pulse are sampled at the the left and the right boundaries of the gain medium and the corresponding Fourier spectra are shown in Fig. 5.

The gain coefficient calculated by the FDTD method using Eq. (3.22) and the theoretical one corresponding to the linear polarization case are plotted in Fig. 6 for rightcircularly-polarized pulse excitation showing an excellent agreement for both $E$ field components.

Finally, we need to prove that the opposite sense of polarization will not deexcite the system. We apply a $\sigma^{-}(+,-)$ left-circularly-polarized pulse at the left boundary of the simulation domain containing gain medium. The results for the gain coefficients are presented in Fig. 7. Thus we have numerically demonstrated selective excitation and deexcita-
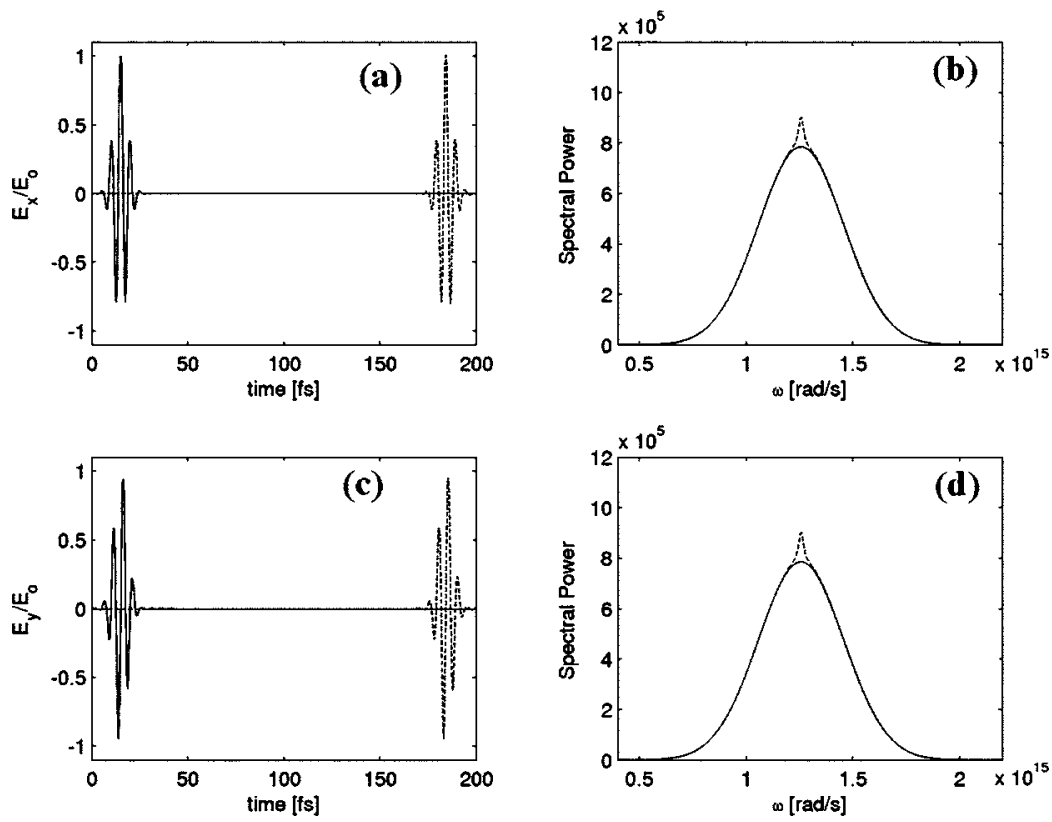

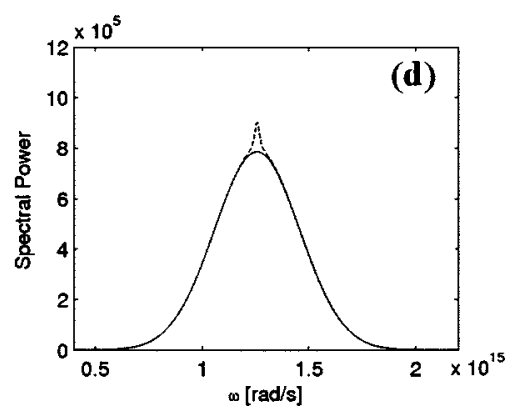

FIG. 5. Time evolution of the $E_{x}$ component (a) and the $E_{y}$ component (c) of a right-circularlypolarized pulse $\sigma^{+}(+,+)$launched in a gain medium (initially pumped into the excited state). The corresponding Fourier spectra (b) and (d) exhibit amplification due to the emission of the absorbed energy by the carriers. 

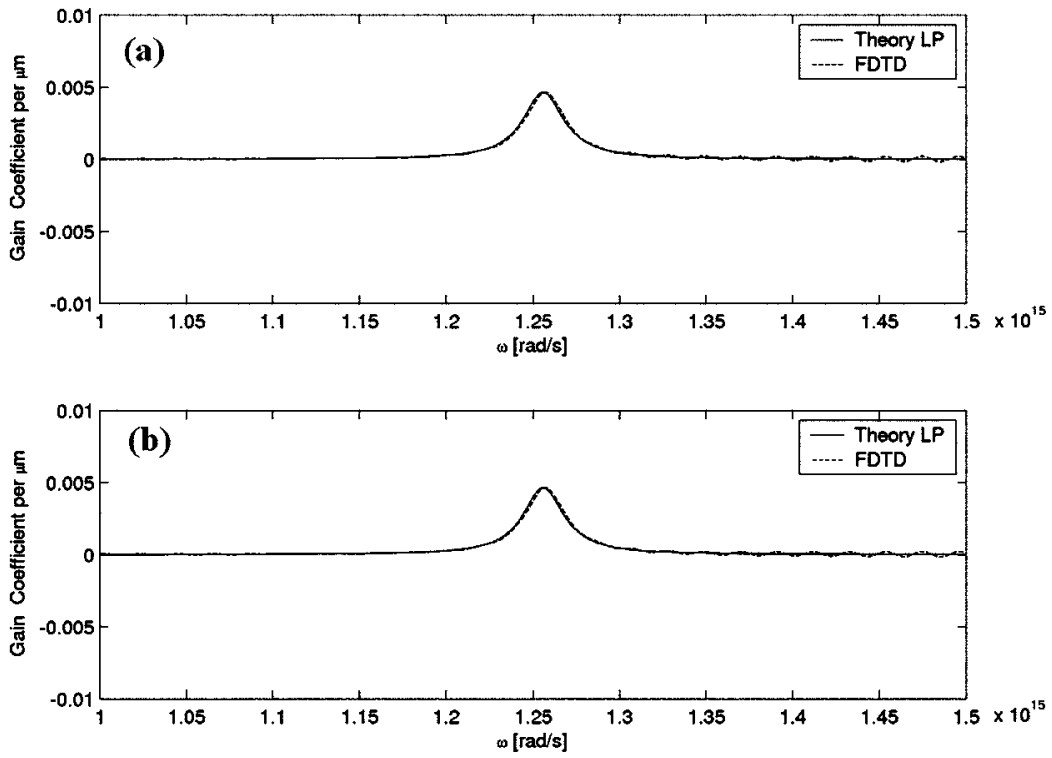

FIG. 6. Gain coefficient calculated by FDTD method for the $E_{x}$ component (a) and the $E_{y}$ component (b) of a right-circularly-polarized pulse $\sigma^{+}(+,+)$compared with the gain coefficient from the linear polarization theory. Excellent agreement between the theory and the FDTD result is achieved. tion of the atomic transition by circularly polarized optical pulses.

\section{SELF-INDUCED TRANSPARENCY AND POLARIZED SOLITON FORMATION}

In this section we investigate the nonlinear regime of polarized pulse propagation which is achieved at high pulse intensities. We shall be interested in the coherent effects, and more specifically, in the self-induced transparency (SIT) which occurs when the ultrashort pulse propagates through media whose atomic lifetimes greatly exceed the pulse duration. Therefore, in order to satisfy the SIT criterion, i.e., $T_{1}$, $T_{2} \gg T_{p}$, we have set $T_{p}=100$ fs and $T_{1}=T_{2}=100$ ps throughout the simulations. The simulation domain is $15 \mu \mathrm{m}$ long and the two-level absorbing medium (corresponding to initial population profile $S_{30}=-1$ ) with refractive index $n=1$ is embedded between two free-space regions each with length $7.5 \mu \mathrm{m}$. As we have shown in the previous Sec. III (Figs. 2 and 3), the only polarized optical wave which excites the system [Fig. 1(a)] initially in its ground state, is the leftcircularly-polarized one: $\sigma^{-}(+,-)$. Therefore we inject a leftcircularly-polarized pulse given by Eq. (3.1) and (3.2) with a hyperbolic secant (HS) envelope given by the first line in Eq. (3.5) at the left boundary $(z=0)$ of the simulation domain. We make an initial guess for the pulse shape to be a HS one since the stable soliton solution of Maxwell-Bloch equations in $1 \mathrm{D}$ is of this form [13]. The carrier frequency is taken to be equal to the resonant transition frequency of the two-level medium $\omega_{0}=2 \pi f_{0}$, where $f_{0}=2.0 \times 10^{14} \mathrm{~s}^{-1}$, corresponding to a wavelength $\lambda=1.5 \mu \mathrm{m}$. According to the pulse area theorem (PAT) which has been formulated within the slowlyvarying-envelope approximation $[14,15]$, the stable solutions in an absorbing medium are given by even multiples of $\pi$. We shall chose the initial field amplitude for both $E_{x}$ and $E_{y}$ components to give a $2 \pi$ pulse area, which yields $E_{0}$ $=4.2186 \times 10^{9} \mathrm{~V} \mathrm{~m}^{-1}[7]$. The results from the simulation are plotted in Fig. 8. It is clear that both pulse components travel
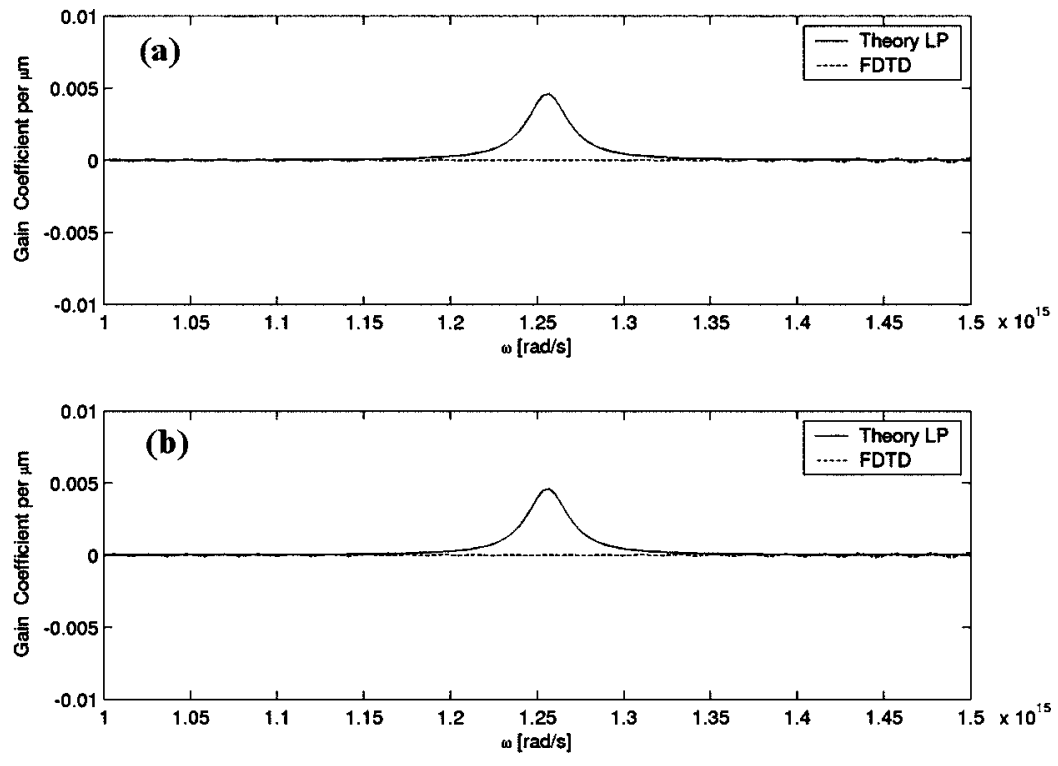

FIG. 7. Gain coefficient calculated by FDTD method for the $E_{x}$ component (a) and the $E_{y}$ component (b) of a left-circularly-polarized pulse $\sigma^{-}(+,-)$plotted together with the gain coefficient from the linear polarization theory showing failure of deexcitation of the two-level system by left-circularly-polarized light. 


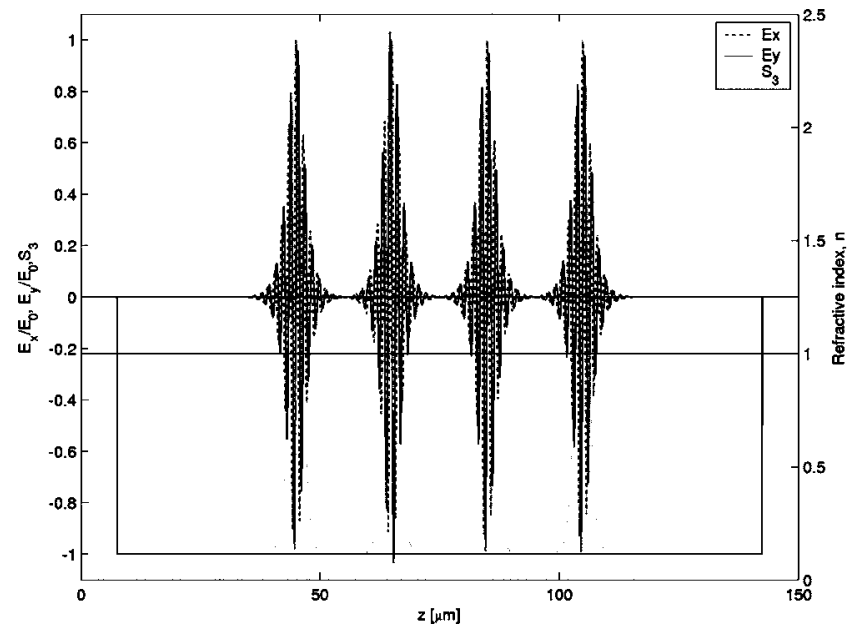

FIG. 8. Time evolution of a $2 \pi$ SIT left-circularly-polarized pulse $\sigma^{-}(+,-)$propagating in absorbing medium (the initial population profile $S_{30}=-1$ shows the boundaries of the absorbing medium). The normalized electric field components $E_{x}$ and $E_{y}$ are plotted together with the population inversion profile $S_{3}$ at the simulation times $t=200,267,334$, and $400 \mathrm{fs}$. The pulse travels undistorted through the two-level medium.

undistorted through the absorbing medium as a solitary wave driving the population inversion through a full Rabi flop. A single pulse at the simulation time $t=200 \mathrm{fs}$ from Fig. 8 is plotted on an expanded scale in Fig. 9 showing the local excitation of the two-level system by the leading pulse edges and the subsequent deexcitation by the trailing pulse edges. This is indicative for the self-induced transparency phenomenon and in agreement with the predictions of the PAT. Thus we have numerically demonstrated the formation of polarized SIT solitons excited by a certain polarization state of the optical field (left-circular polarization in this case). Note that the cubic-polynomial features characteristic for the linear po-

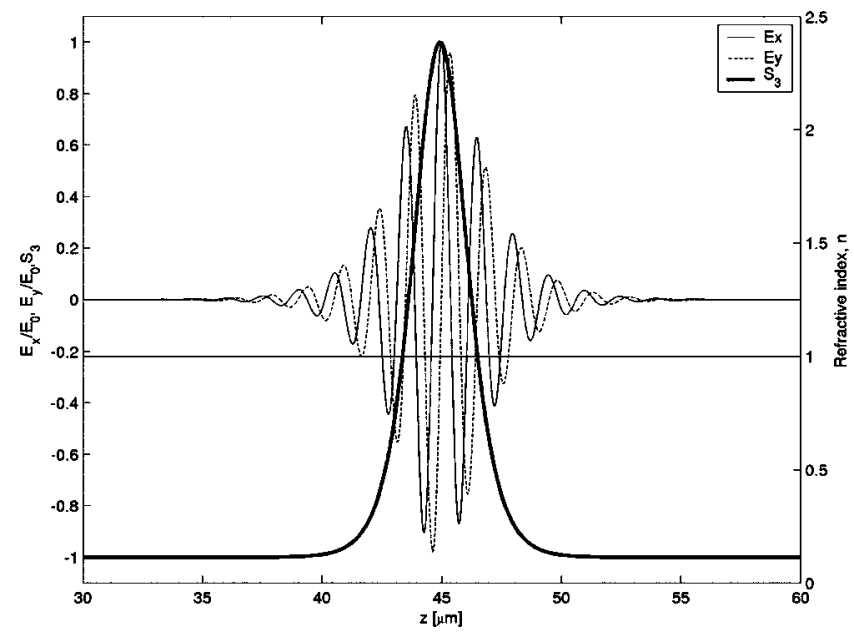

FIG. 9. Left-circularly-polarized $2 \pi$ SIT pulse $\sigma^{-}(+,-)$with a hyperbolic secant envelope at the simulation time $t=200 \mathrm{fs}$. The normalized $E$ field components are plotted together with the population inversion. The polarized SIT pulse completely inverts locally the two-level medium and returns the population back to the ground state within one Rabi flop.

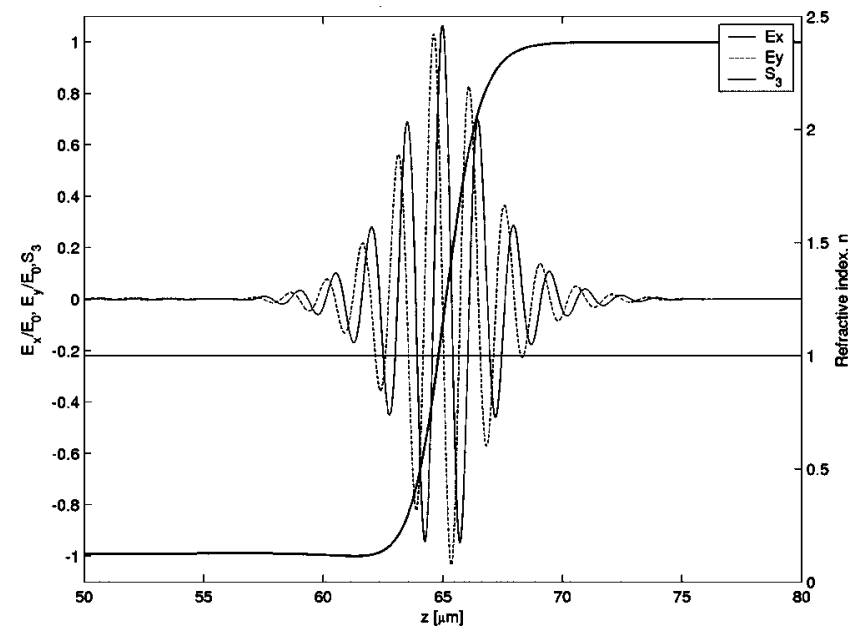

FIG. 10. Right-circularly-polarized SIT $\pi$ pulse $\sigma^{+}(+,+)$completely inverts the medium returning the population back to the ground state. The normalized $E$ field components $\left(E_{x, y}\right)$ and the population $\left(S_{3}\right)$ are given at the simulation time $t=267 \mathrm{fs}$.

larization case occurring at the null-field points attributed to the time-derivative behavior [7] are no longer present in the circularly polarized case (Fig. 9). This is due to the superposition of the effects of the time derivatives of the $E$ field components, the second being time shifted with respect to the first one.

To complete our validation study we need to check the predictions of the PAT regarding the polarized pulse propagation in an amplifying (gain) two-level media. By the virtue of the PAT, the stable solutions are obtained when the pulse area is odd multiples of $\pi$. The simulation domain is as described above except for the population which is assumed to be initially in the excited level, corresponding to $S_{30}=+1$. We launch a $\pi$ right-circularly-polarized pulse $\sigma^{+}(+,+)$from the left boundary, given by Eqs. (3.3) and (3.4), modulated by a HS function (3.5). The initial pulse amplitude is taken $E_{0}=2.1093 \times 10^{9} \mathrm{~V} \mathrm{~m}^{-1}$ which corresponds to pulse area of $\pi$. The pulse and the corresponding population inversion are plotted in Fig. 10. As expected from the PAT, the pulse completely inverts the medium returning the population back to the ground state. The pulse time evolution is shown in Fig. 11. The pulse amplitude increases while the pulse duration is shortened. As a result the pulse area is conserved during the propagation in the amplifying medium.

\section{CONCLUSIONS}

We have developed a formalism and a numerical method based on it for the exact treatment of polarized ultrashort optical pulse interactions with a model two-level quantum system. The proposed approach takes advantage of the real pseudospin vector representation in its little known ramification for description of the time evolution of a two-level quantum system in case of excitation by circularly polarized light due to Feynman et al. [1]. This in turn allows for a coupling to the vectorial Maxwell equations in real space (using real electric field components rather than complex 


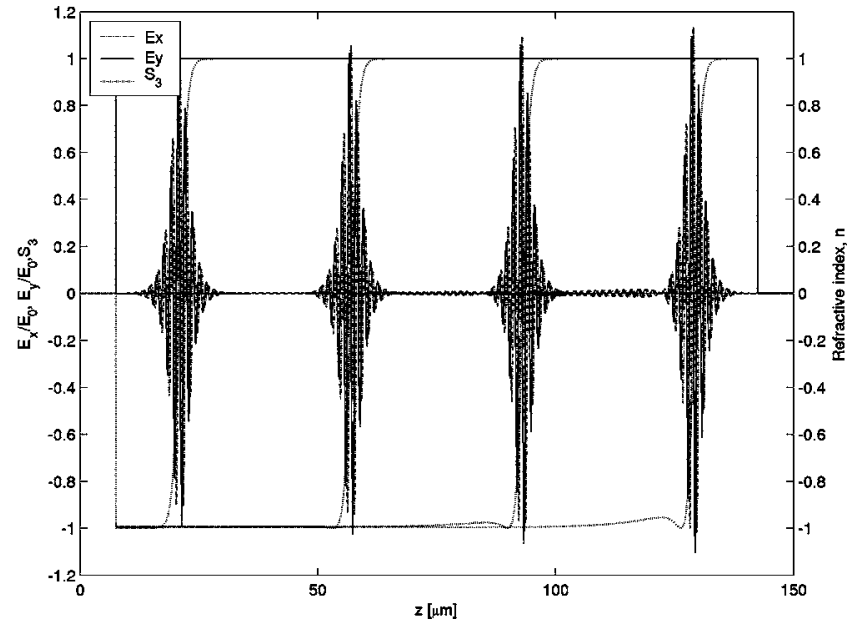

FIG. 11. Time evolution of a right-circularly-polarized SIT $\pi$ pulse $\sigma^{+}(+,+)$. The normalized $E$ field components $\left(E_{x, y}\right)$ and the population $\left(S_{3}\right)$ are given at the simulation time $t$ $=120,240,360,480 \mathrm{fs}$.

ones) and for a direct FDTD solution in the time domain without invoking any approximations such as the SVEA and RWA. This permits for a rigorous description of pulse propa- gation and interactions on an ultrashort time scale including few-optical-cycle pulses which are the most likely candidates to be used for realization of coherent quantum control of spin-polarized states. The proposed model fully accounts for the vector character of the electric field of the ultrafast optical excitation and hence for its polarization state. We demonstrated selective excitation of atomic transitions with $\Delta J_{z}$ $=1$ or $\Delta J_{z}=-1$ by predefined helicity of the optical pulse. Another important advantage of the FDTD solution of the Maxwell pseudospin system is the possibility of treatment of a variety of realistic geometries including microcavities (see $[5,6])$. The range of validity of the model potentially reaches to the extreme nonlinear regime. It is successfully applied to the study of coherent propagation phenomena, such as SIT, and the formation of polarized solitons is numerically demonstrated. The model is currently being extended to multiple dimensions and multilevel quantum systems.

\section{ACKNOWLEDGMENTS}

The authors gratefully acknowledge the CPU time on the parallel cluster available at the ATI. We wish to thank K. Boehringer and A. Klaedtke for helpful and elucidating discussions which stimulated the present work.
[1] R. P. Feynman, F. L. Vernon, and R. W. Hellwarth, J. Appl. Phys. 28, 49 (1957).

[2] M. Kroutvar, Y. Ducommun, D. Heiss, M. Bichler, D. Schuh, G. Abstreiter, and J. Finley, Nature (London) 432, 81 (2004).

[3] G. Slavcheva, J. M. Arnold, I. Wallace, and R. W. Ziolkowski, Phys. Rev. A 66, 063418 (2002).

[4] H. Haug and S. W. Koch, Quantum Theory of the Optical and Electronic Properties of Semiconductors, 3rd ed. (World Scientific, Singapore, 1994), p. 79.

[5] G. Slavcheva, J. M. Arnold, and R. W. Ziolkowski, IEEE J. Sel. Top. Quantum Electron. 9, 929 (2003).

[6] G. Slavcheva, J. M. Arnold, and R. W. Ziolkowski, IEEE J. Sel. Top. Quantum Electron. 10, 1052 (2004), (special issue on nonlinear optics).

[7] R. W. Ziolkowski, J. M. Arnold, and D. M. Gogny, Phys. Rev.
A 52, 3082 (1995).

[8] F. T. Hioe and J. H. Eberly, Phys. Rev. Lett. 47, 838 (1981).

[9] F. T. Hioe, Phys. Rev. A 28, 879 (1983).

[10] A. C. Newell and J. V. Moloney, Nonlinear Optics (AddisonWesley, Redwood City, CA, 1992), p. 233.

[11] A. Taflove, Computational Electrodynamics: The FiniteDifference Time-Domain Method (Artech, Norwood, MA, 1995).

[12] A. Yariv, Quantum Electronics (Wiley, New York, 1989), p. 159.

[13] L. Allen and J. H. Eberly, Optical Resonance and Two-Level Atoms (Wiley, New York, 1975).

[14] S. L. McCall and E. L. Hahn, Phys. Rev. Lett. 18, 908 (1967).

[15] S. L. McCall and E. L. Hahn, Phys. Rev. 183, 457 (1969). 